\title{
Quest for Soft X-ray Tomographic Ptychography using KB focusing Optics
}

\author{
$\underline{\text { Hung Wei Shiu }}^{1}$, Yen-Yi Chu ${ }^{1}$, Lo-Yueh Chang ${ }^{2,1}$, Singh Amol ${ }^{1}$, Tolek Tyliszczak ${ }^{1}$ and Di-Jing \\ Huang, \\ 1. National Synchrotron Radiation Research Center, Hsinchu, Taiwan. \\ 2. Institute of Functional Nano \& Soft Materials (FUNSON), Soochow University, Soochow, China. \\ *djhuang@nsrrc.org.tw.
}

Soft X-ray microscopy is a powerful technique which can probe materials at a good spatial resolution with chemical, electronic and orientation contrast [1]. Recently, based on scanning transmission X-ray microscopy (STXM), soft X-ray ptychography — scanning coherent diffraction imaging — has been developed to dramatically improve the spatial resolution and spectral information [2]. This advent can, in principle, achieve a spatial resolution beyond the limitation of X-ray lenses. It is much in demand to extend the 2D ptychography to a 3D imaging through a tomographic scheme. However, the realization of soft X-ray tomographic ptychography is challenging because of the limited working distance of zoneplate optics used in STXM.

In this presentation, we show a feasibility of realizing soft X-ray tomographic ptychography by using Kirkpartrick-Baes (KB) optics. Soft X-rays with a sufficient coherent flux were extracted from an undulator beamline at the Taiwan Photon Source (TPS). With proper optics and filtering, the coherent flux from the elliptically polarizing undulator (EPU) source for energy between 400 and $1200 \mathrm{eV}$ is in the range of $10^{11}$ photons/s. The monochromatic beam was generated by an active grating monochromator (AGM) and focused onto an exit slit which serves as a secondary source. A resolving power higher than 4500 is achieved for energy below $900 \mathrm{eV}$ if the exit slit opening is set to $30 \mu \mathrm{m}$. The incoherent portion of the beam outside of the coherent angle of $\lambda / d$, where $d$ is the slit opening, was rejected by a set of the coherent slits in both horizontal and vertical directions.

The layout and the photo of the soft X-ray ptychography endstation are shown in Fig. 1. The monochromatic light from the secondary source is refocused onto the sample by the KB optics, which provide a high intensity of focused beam and enable a large working distance. In our design, the distance between the KB optics and the sample is 2 meter, where a set of spatial filters in horizontal and vertical direction are placed to clean up the coherent beam and to minimize the influence of the optics. With 2D sample scanning stages of high spatial precision, the probe area can be precisely controlled. For the STXM mode, the intensity of transmitted light is directly collected by a retractable avalanche photodiode, while a CCD detector is used to record the speckle pattern in the ptychography mode. We used attenuators of three different thickness placed in front of the CCD to reduce the intensity of the direct beam and to avoid the bleeding effect of the saturation signal, expanding the dynamic range of the CCD. In this geometry, the resolution limit for $850 \mathrm{eV}$ is $4 \mathrm{~nm}$ if a CCD with a frame size $27.6 \mathrm{~mm}$ and a pixel size $13.5 \mu \mathrm{m}$ is used and its distance to the sample is $70 \mathrm{~mm}$.

The measured beamsize at the sample position is $\sim 8 \mu \mathrm{m}$ in both horizontal and vertical directions. Although the spatial resolution of STXM images obtained by using this beamsize is coarse, it is sufficient for us to identify the region of interest. Figure 2 shows the first preliminary speckle pattern obtained at $650 \mathrm{eV}$ with a $2 \mu \mathrm{m}$ pinhole placed at $1 \mathrm{~mm}$ before the sample. The sample was a 250-nmthick Au test NSRRC logo of high resolution, as shown in the inset of Fig. 2 in which a red open circle 
indicates the illuminated area. The triangle shadow and the black line in the speckle pattern are the images of an aluminium attenuator and its supporting wire, respectively. The attenuator was used to reduce the intensity of the straight light. A preliminary reconstruction pattern was successfully achieved; however the result is not perfect (not shown here). Ptychography taken with pinholes of various sizes is under test in order to achieve a good reconstruction.

In summary, we have successfully constructed a soft X-ray ptychography microscope and obtained the first speckle pattern at TPS. Although the image of the first reconstruction was not perfect, it is a good starting point for us to develop a microscope of temperature-dependent soft X-ray tomographic ptychography. Currently we are using a CCD camera, which has a pixel readout rate of $1 \mathrm{MHz}$. In the future we will upgrade our system to have a new fast CCD or CMOS camera and extend our capabilities to low temperatures for tomography applications by combining a commercial liquid-helium cryostat, low-temperature piezo stages and in-vacuum diffractometer. A KB-like optic, a capillary focusing optic, will also be used to further reduce the beamsize to be smaller than $200 \mathrm{~nm}$ at $650 \mathrm{eV}$ and to have a relative large working distance $(\sim 5 \mathrm{~mm})$.

\section{References:}

[1] A. P. Hitchcock, J. Electron. Spectrosc. Relat. Phenom. 200, (2015), 49.

[2] D. A. Shapiro et al, Nat. Photon. 8, (2014), 765.

[3] Y. Takahashi et al, Phys. Rev. B 83, (2011), 214109.
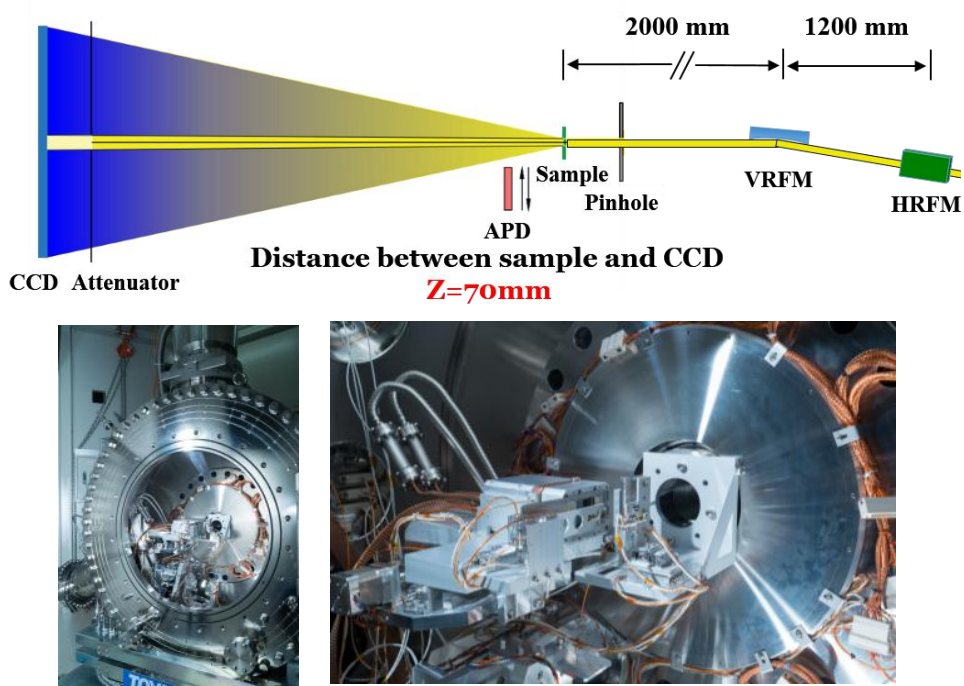

Figure. 1. Optical layout and photos of the ptychography endstation. The circular chamber shown in the bottom left is a diffractometer system for resonance scattering and Bragg CDI. An enlarged picture of the ptychography microscope is shown in the bottom right.

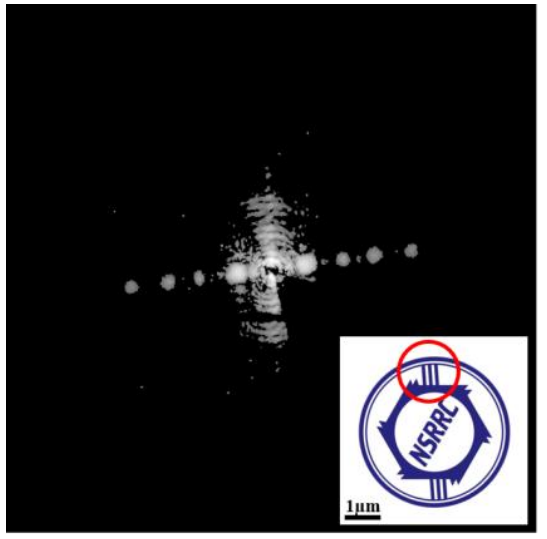

Figure. 2. Log-scale speckle pattern of a Au test object. The frame size of this image is $400 \mathrm{x} 400$ pixels. The inset shows the design pattern and illumination area. A $2-\mu \mathrm{m}$ pinhole was placed at 1 $\mathrm{mm}$ before the test object. 